\title{
Segregation of $\mathrm{Nb}$ to $\mathrm{TiO}_{2}$ Grain Boundaries
}

\author{
Ying Pang*, Noel T.Nuhfer* and Paul Wynblatt* \\ * Department of Materials Science and Engineering, Carnegie Mellon University, PA 15213, USA
}

Considerable effort has been devoted to the study of solute or impurity segregation at grain boundaries over the past several decades. Although there has been some previous research on grain boundary segregation as a function of grain boundary misorientation, only special grain boundaries, and limited numbers of general grain boundaries, have been examined [1]. In this study, we performed measurements in a scanning Auger microprobe (SAM) of $\mathrm{Nb}$ segregation to $\mathrm{TiO}_{2}$ grain boundaries on 83 boundaries exposed by intergranular fracture, in a sample of $2 \mathrm{~mol} \% \mathrm{Nb}$-doped $\mathrm{TiO}_{2}$. Auger compositional information was obtained on both fracture surfaces of each grain boundary. In addition, the orientation of each grain boundary fracture plane was determined by combining a stereo-pair method implemented in a scanning electron microscope (SEM) with orientation imaging microscopy (OIM) [2]. In Fig. 1, the crystallographic orientation of the grain boundary surface planes, from both sides of the fracture surface (total of 166 measurements) are plotted in the standard stereographic triangle for the tetragonal crystal system. It indicates that the degree of $\mathrm{Nb}$ segregation correlates with the crystallographic orientation of the planes which bound the crystal on either side of the grain boundary. Fig.2 shows the surface energies of the corresponding crystal planes from theoretical estimates. These two results suggest that segregation is stronger at boundaries comprised of high energy surface planes than boundaries comprised of low energy surface planes [3].

Whereas AES is a useful technique for the study of grain boundary segregation, and its implementation in a SAM allows measurements on several tens of boundaries in reasonable times, there are two aspects of the results that can usefully be complemented by higher resolution techniques such as HRTEM. The first is related to possible artifacts in the AES results that can arise from the presence of unresolved second phases at the grain boundaries. If such phases are present but undetected, then high grain boundary concentrations of solute due to these phases could be misinterpreted as grain boundary segregation. We have examined thin sections of the $\mathrm{Nb}$-doped $\mathrm{TiO}_{2}$ by HRTEM. A typical grain boundary image is displayed in Fig. 3(a). The figure shows that the grain boundaries are free of precipitates and of amorphous phase films (frequently present in oxide systems). The second aspect of the results that cannot readily be answered by AES is the issue of grain boundary equilibration. If solute must diffuse from the bulk of the grains to the grain boundary regions, in order to bring the boundaries up to their equilibrium segregation level, there will be a transient period during which solute depletion can be seen near boundaries. Absence of a solute-depleted region thus provides good evidence for equilibrated boundaries. The spatial distribution of the $\mathrm{Nb}$ segregation region from EDXS with the STEM HAADF image (Fig.3b) indicates that the segregation layer is about $4 \mathrm{~nm}$ and that no depleted region is present.

\section{$\underline{\text { References }}$}

[1] S.S. Brenner, M-J. Hua, Scripta Metall., 24, 667 (1990).

[2] Y. Pang, P. Wynblatt, J.Amer.Ceram.Soc., to be published.

[3] Y.Pang, P.Wynblatt, Acta Mater, to be published.

[4] The authors wish to acknowledge with thanks support of this research by the MRSEC Program of the National Science Foundation under award number DMR-0079996. 

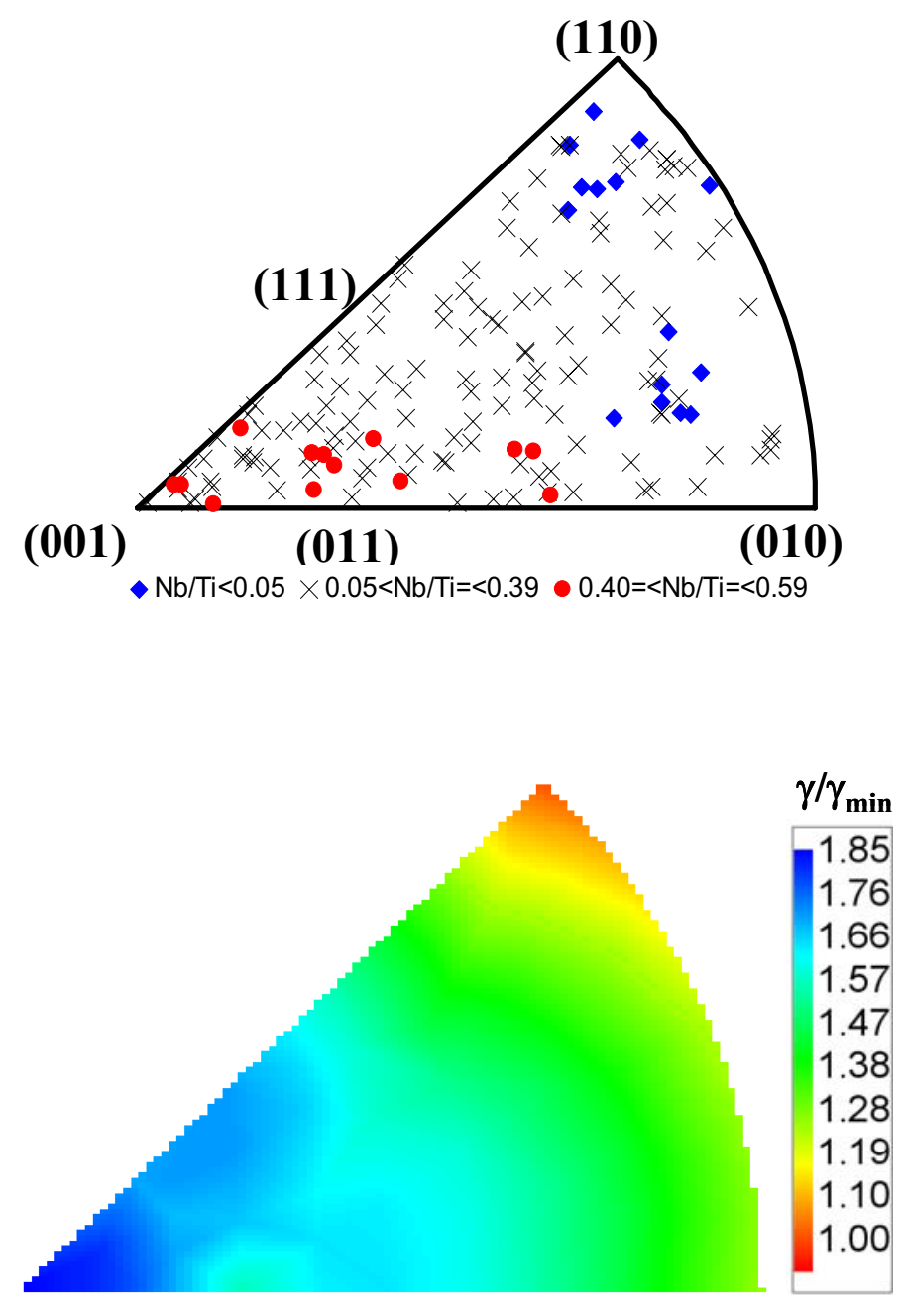

Fig.1. Variation of Auger $\mathrm{Nb} / \mathrm{Ti}$ peak ratios with orientation of the grain boundary plane, obtained from intergranular fracture of $2 \mathrm{~mol} \% \mathrm{Nb}$ $\mathrm{TiO}_{2}$. (Relative segregation level is sorted into three groups identified by lozenges for low $\mathrm{Nb} / \mathrm{Ti}$ ratio, crosses for moderate $\mathrm{Nb} / \mathrm{Ti}$ ratio, and solid circles for high $\mathrm{Nb} / \mathrm{Ti}$ ratio.)

Fig.2 Surface energy anisotropy of $\mathrm{TiO}_{2}$ plotted in a standard stereographic triangle for a tetragonal crystal, for comparison with grain boundary compositions of Fig.1. (Red color represents the lowest surface energy, and blue color represents the highest surface energy.)

Fig.3. (a) High-resolution TEM image showing clean grain boundary without amorphous phase in $2 \mathrm{~mol} \% \mathrm{Nb}-\mathrm{TiO}_{2}$; (b) EDXS file taken across the grain boundary with inset STEM HAADF image.
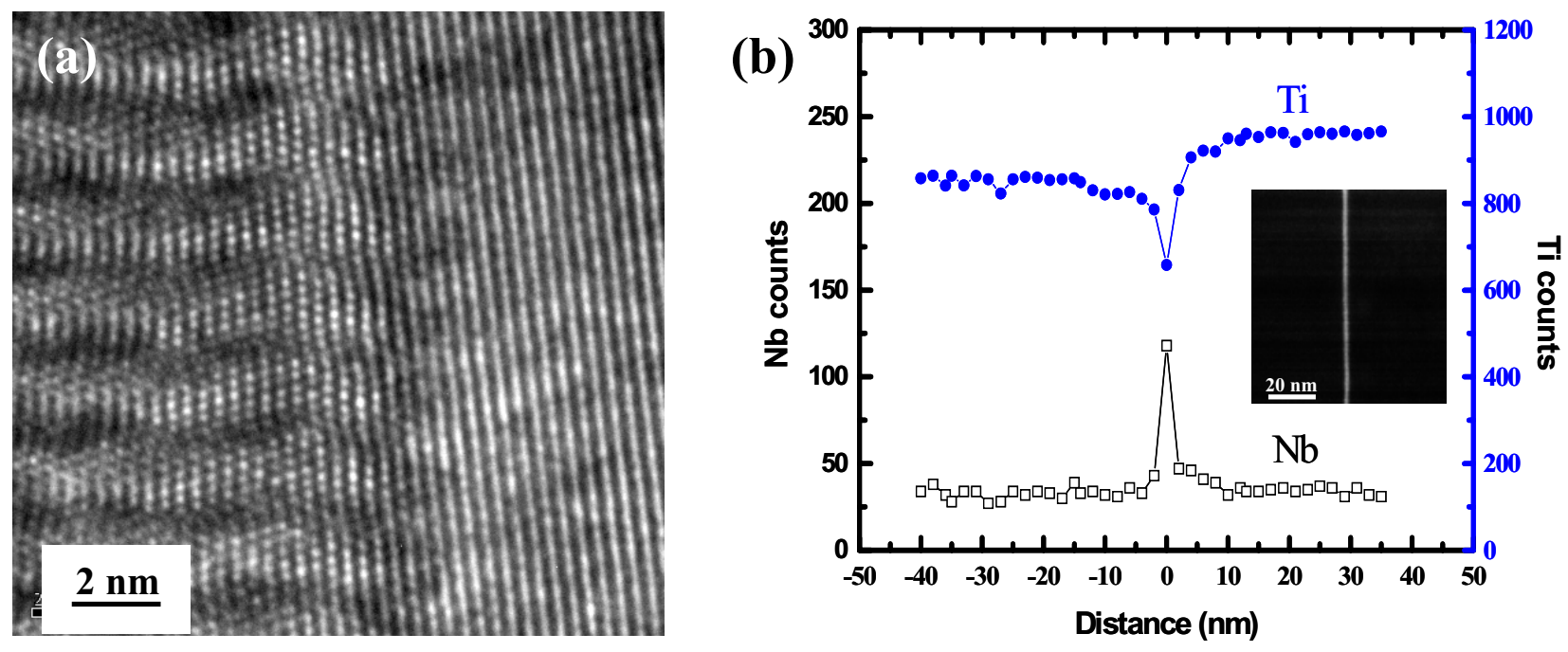